\title{
Noise Suppresses or Expresses Exponential Growth
}

\author{
Feiqi Deng ${ }^{1}$, Qi Luo ${ }^{2}$, Xuerong Mao $^{3, *}$, Sulin Pang ${ }^{4}$ \\ ${ }^{1}$ Systems Engineering Institute, \\ South China University of Technology, Guangzhou 510640, China \\ ${ }^{2}$ Department of Information and Communication, \\ Nanjing University of Information Science and Technology \\ Nanjing 210044, P.R. China \\ ${ }^{3}$ Department of Statistics and Modelling Science, \\ University of Strathclyde, Glasgow G1 1XH, U.K. \\ ${ }^{4}$ Department of Accountancy \& Institute of Finance Engineering, \\ School of Management, Jinan University, \\ Guangzhou 510632, P.R. China
}

\begin{abstract}
In this paper we will show that noise can make a given system whose solutions grow exponentially become a new system whose solutions will grow at most polynomially. On the other hand, we will also show that noise can make a given system whose solutions are bounded become a new system whose solutions will grow exponentially. In other words, we reveal that the noise can suppress or expresses exponential growth.
\end{abstract}

Key words: Brownian motion, stochastic differential equation, stochastic control, exponential growth, polynomial growth, boundedness.

AMS Classifications: $60 \mathrm{H} 10,93 \mathrm{E} 15$

\section{Introduction}

It is well known that noise can be used to stabilise a given unstable system or to make a system even more stable when it is already stable. There is an extensive literature concerned with the stabilisation and destabilisation by noise and we here mention $[1,2$, 3, 4, 5, 7, 8, 9, 10, 11, 14, 18, 20, 21]. Recently, Mao et al. [16] showed another important fact that the environmental noise can suppress explosions (in a finite time) in population

*Corresponding author. E-mail: xuerong@stams.strath.ac.uk 
dynamics. In this paper we will reveal one more important feature that the noise can suppress or expresses exponential growth.

To explain this feature more clearly, let us consider the simple linear scalar ordinary differential equation

$$
\dot{y}(t)=a+b y(t) \quad \text { on } t \geq 0
$$

with initial value $y(0)=y_{0}>0$, where $a, b>0$. This equation has its explicit solution

$$
y(t)=\left(y_{0}+\frac{a}{b}\right) e^{b t}-\frac{a}{b} .
$$

Hence

$$
\lim _{t \rightarrow \infty} \frac{1}{t} \log (y(t))=b,
$$

that is, the solution tends to infinity exponentially. On the other hand, we stochastically perturb this equation into a linear stochastic differential equation (SDE)

$$
d x(t)=[a+b x(t)] d t+\sigma x(t) d B(t) \quad \text { on } t \geq 0,
$$

where $\sigma>0$ and $B(t)$ is a scalar Brownian motion. Given initial value $x(0)=x_{0}>0$, this SDE has its explicit solution

$$
x(t)=\exp \left[\left(b-\frac{1}{2} \sigma^{2}\right) t+\sigma B(t)\right]\left(x_{0}+a \int_{0}^{t} \exp \left[\left(b-\frac{1}{2} \sigma^{2}\right) s+\sigma B(s)\right] d s\right) .
$$

We shall see later in this paper that if $\sigma^{2}>2 b$ then the solution of equation (1.2) obeys

$$
\limsup _{t \rightarrow \infty} \frac{\log (x(t))}{\log t} \leq \frac{\sigma^{2}}{\sigma^{2}-2 b} \quad \text { a.s. }
$$

This shows that for any $\varepsilon>0$, there is a positive random variable $T_{\varepsilon}$ such that, with probability one,

$$
x(t) \leq t^{\varepsilon+\sigma^{2} /\left(\sigma^{2}-2 b\right)} \quad \forall t \geq T_{\varepsilon} .
$$

In other words, the solution will grow at most polynomially with order $\varepsilon+\sigma^{2} /\left(\sigma^{2}-2 b\right)$. In particular, by increasing the noise intensity $\sigma$, we can make the order as closed to 1 as we like. Comparing this polynomial growth with the exponential growth of the solution (1.1), we see the important fact that the noise suppresses the exponential growth.

We should also point out another important feature. As the noise term in equation (1.2) is linear, the mean value $\mathbb{E} x(t)$ of the solution still obeys its original equation (1.1) so $\mathbb{E} x(t)$ will tend to infinity exponentially. This is significantly different from its sample property that almost every sample path of the solution will grow at most polynomially. The classical relationship between almost sure and moment Lyapunov exponents (see e.g. $[6,19])$ illustrates this issue more clearly.

The main aim of this paper is to develop this idea for general nonlinear SDEs. We will then consider a nonlinear system described by an ordinary differential equation

$$
\dot{y}(t)=f(y(t), t),
$$

whose coefficient obeys

$$
\langle y, f(y, t)\rangle \leq K_{1}+K_{2}|y|^{2}, \quad \forall(y, t) \in \mathbb{R}^{n} \times \mathbb{R}_{+} .
$$


Clearly, the solution of this equation may grow exponentially. However, we will show that we can always design a linear stochastic feedback control $A x(t) d B(t)$ (i.e. choose a square matrix $\left.A \in \mathbb{R}^{n \times n}\right)$ so that the stochastically controlled system

$$
d x(t)=f(x(t), t) d t+A x(t) d B(t)
$$

will grow at most polynomially with probability one.

However, every thing has two sides. We may therefore wonder if noise can make a given system whose solutions are bounded become a new system whose solutions will grow exponentially? In this paper we will give a positive answer to this question as well. For example, the $n$-dimensional linear ordinary differential equation $\dot{y}(t)=q+Q y(t)$ has bounded solutions if the square matrix $Q$ is negative-definite. If $n$ is an even number, we can perturb it by two independent Brownian motions into a linear SDE

$$
d x(t)=(q+Q x(t)) d t+\xi d B_{1}(t)+A x(t) d B_{2}(t)
$$

so that its solutions will grow exponentially with probability one. It is also interesting to observe that its mean values $\mathbb{E} x(t)$ still obey the original equation $\dot{y}(t)=q+Q y(t)$ whence they are still bounded.

\section{Polynomial Growth of SDEs}

Throughout the paper, unless otherwise specified, we will employ the following notation. Let $\left(\Omega, \mathcal{F},\{\mathcal{F}(t)\}_{t \geq 0}, \mathbb{P}\right)$ be a complete probability space with a filtration $\{\mathcal{F}(t)\}_{t \geq 0}$ satisfying the usual conditions (i.e., it is increasing and right continuous while $\mathcal{F}(0)$ contains all $\mathbb{P}$-null sets). Let $B(t)=\left(B_{1}(t), \cdots, B_{m}(t)\right)^{T}, t \geq 0$, be an $m$-dimensional Brownian motion defined on the probability space, where $T$ denotes the transpose of a vector or matrix. If $x, y$ are real numbers, then $x \vee y$ denotes the maximum of $x$ and $y$, and $x \wedge y$ denotes the minimum of $x$ and $y$. Let $|x|$ be the Euclidean norm of a vector $x \in \mathbb{R}^{n}$ and $\langle x, y\rangle$ be the inner product of vectors $x, y \in \mathbb{R}^{n}$. Vectors $x \in \mathbb{R}^{n}$ are thought as column ones so to get row vectors we use $x^{T}$. The space of $n \times m$ matrices with real entries is denoted by $\mathbb{R}^{n \times m}$. If $A=\left(a_{i j}\right)$ is an $n \times m$ matrix, we denote its Frobenius or trace norm by

$$
|A|=\sqrt{\sum_{i=1}^{n} \sum_{j=1}^{m} a_{i j}^{2}}
$$

while its operator norm by $\|A\|=\sup \left\{|A x|: x \in \mathbb{R}^{m},|x|=1\right\}$. If $A \in \mathbb{R}^{n \times n}$ is symmetric, its largest and smallest eigenvalue are denoted by $\lambda_{\max }(A)$ and $\lambda_{\min }(A)$, respectively.

Let us consider an $n$-dimensional stochastic differential equation (SDE)

$$
d x(t)=f(x(t), t) d t+g(x(t), t) d B(t)
$$

on $t \geq 0$ with the initial value $x(0)=x_{0} \in \mathbb{R}^{n}$, where

$$
f: \mathbb{R}^{n} \times \mathbb{R}_{+} \rightarrow \mathbb{R}^{n} \quad \text { and } \quad g: \mathbb{R}^{n} \times \mathbb{R}_{+} \rightarrow \mathbb{R}^{n \times m} .
$$

We impose the following hypothesis. 
Assumption 2.1 Assume that both coefficients $f$ and $g$ are locally Lipschitz continuous, that is, for each $k=1,2, \cdots$, there is a positive number $H_{k}$ such that

$$
|f(x, t)-f(y, t)| \vee|g(x, t)-g(y, t)| \leq H_{k}|x-y|
$$

for all $t \geq 0$ and those $x, y \in \mathbb{R}^{n}$ with $|x| \vee|y| \leq k$. Assume also that there are nonnegative constants $\alpha, \beta, \eta$ and $\gamma$ such that

$$
\langle x, f(x, t)\rangle \leq \alpha+\beta|x|^{2}, \quad|g(x, t)|^{2} \leq \eta+\gamma|x|^{2}
$$

for all $(x, t) \in \mathbb{R}^{n} \times \mathbb{R}_{+}$.

It is known (see e.g. [15, Theorem 3.5 on page 58]) that under Assumption 2.1, the $\operatorname{SDE}(2.1)$ has a unique global solution $x(t)$ on $t \in \mathbb{R}_{+}$. We also observe from $[15$, Theorem 5.1 on page 63] that under Assumption 2.1 the solution obeys

$$
\limsup _{t \rightarrow \infty} \frac{1}{t} \log (|x(t)|) \leq \beta+\frac{1}{2} \gamma \quad \text { a.s. }
$$

That is, the solution will grow at most exponentially with probability one. The following theorem shows that if the noise is sufficiently large, it will suppress this potentially exponential growth and make the solution grow at most polynomially.

Theorem 2.2 Let Assumption 2.1 hold. Assume that there are moreover two positive constants $\delta$ and $\rho$ such that

$$
\left|x^{T} g(x, t)\right|^{2} \geq \delta|x|^{4}-\rho
$$

for all $(x, t) \in \mathbb{R}^{n} \times \mathbb{R}_{+}$. If

$$
\delta>\beta+\frac{1}{2} \gamma
$$

then the solution of equation (2.1) obeys

$$
\limsup _{t \rightarrow \infty} \frac{\log (|x(t)|)}{\log t} \leq \frac{\delta}{2 \delta-2 \beta-\gamma} \quad \text { a.s. }
$$

Proof. Choose any $\theta$ such that

$$
0<\theta<\frac{2 \delta-2 \beta-\gamma}{2 \delta} .
$$

By the Itô formula,

$$
\begin{aligned}
d\left[\left(1+|x(t)|^{2}\right)^{\theta}\right] & =\left(\theta\left(1+|x(t)|^{2}\right)^{\theta-1}\left[2\langle x(t), f(x(t), t)\rangle+|g(x(t), t)|^{2}\right]\right. \\
& \left.+2 \theta(\theta-1)\left(1+|x(t)|^{2}\right)^{\theta-2}\left|x^{T}(t) g(x(t), t)\right|^{2}\right) d t \\
& +2 \theta\left(1+|x(t)|^{2}\right)^{\theta-1} x^{T}(t) g(x(t), t) d B(t) .
\end{aligned}
$$


By conditions (2.2) and (2.3), we then have

$$
\begin{aligned}
d\left[\left(1+|x(t)|^{2}\right)^{\theta}\right] \leq & \theta\left(1+|x(t)|^{2}\right)^{\theta-2} \\
\times & {\left[\left(1+|x(t)|^{2}\right)\left[2 \alpha+\eta+(2 \beta+\gamma)|x(t)|^{2}\right]-2(1-\theta)\left(\delta|x(t)|^{4}-\rho\right)\right] d t } \\
+ & 2 \theta\left(1+|x(t)|^{2}\right)^{\theta-1} x^{T}(t) g(x(t), t) d B(t) \\
= & \theta\left(1+|x(t)|^{2}\right)^{\theta-2}\left(2 \alpha+\eta+2 \rho+(2 \alpha+\eta+2 \beta+\gamma)|x(t)|^{2}\right. \\
& \left.\quad-[2 \delta(1-\theta)-2 \beta-\gamma]|x(t)|^{4}\right) d t \\
+ & 2 \theta\left(1+|x(t)|^{2}\right)^{\theta-1} x^{T}(t) g(x(t), t) d B(t) .
\end{aligned}
$$

Choose $\varepsilon>0$ sufficiently small for

$$
\frac{\varepsilon}{\theta}<2 \delta(1-\theta)-2 \beta-\gamma
$$

Then, by the Itô formula again,

$$
\begin{aligned}
& d\left[e^{\varepsilon t}\left(1+|x(t)|^{2}\right)^{\theta}\right] \\
\leq & \theta e^{\varepsilon t}\left(1+|x(t)|^{2}\right)^{\theta-2}\left(\frac{\varepsilon}{\theta}\left(1+|x(t)|^{2}\right)^{2}+2 \alpha+\eta+2 \rho\right. \\
& \left.+(2 \alpha+\eta+2 \beta+\gamma)|x(t)|^{2}-[2 \delta(1-\theta)-2 \beta-\gamma]|x(t)|^{4}\right) d t \\
+ & 2 \theta e^{\varepsilon t}\left(1+|x(t)|^{2}\right)^{\theta-1} x^{T}(t) g(x(t), t) d B(t) .
\end{aligned}
$$

It is easy to see that there is a positive constant $C_{1}$ such that

$$
\begin{aligned}
\theta & \left(1+|x|^{2}\right)^{\theta-2}\left(\frac{\varepsilon}{\theta}\left(1+|x|^{2}\right)^{2}+2 \alpha+\eta+2 \rho\right. \\
& \left.+(2 \alpha+\eta+2 \beta+\gamma)|x|^{2}-[2 \delta(1-\theta)-2 \beta-\gamma]|x|^{4}\right) \leq C_{1}
\end{aligned}
$$

for all $x \in \mathbb{R}^{n}$. Thus

$$
d\left[e^{\varepsilon t}\left(1+|x(t)|^{2}\right)^{\theta}\right] \leq C_{1} e^{\varepsilon t} d t+2 \theta e^{\varepsilon t}\left(1+|x(t)|^{2}\right)^{\theta-1} x^{T}(t) g(x(t), t) d B(t) .
$$

This implies

$$
\mathbb{E}\left[e^{\varepsilon t}\left(1+|x(t)|^{2}\right)^{\theta}\right] \leq\left[\left(1+\left|x_{0}\right|^{2}\right)^{\theta}\right]+\frac{C_{1}}{\varepsilon} e^{\varepsilon t},
$$

whence

$$
\limsup _{t \rightarrow \infty} \mathbb{E}\left[\left(1+|x(t)|^{2}\right)^{\theta}\right] \leq \frac{C_{1}}{\varepsilon} .
$$

Moreover, using (2.9), we observe from (2.7) that

$$
d\left[\left(1+|x(t)|^{2}\right)^{\theta}\right] \leq C_{1} d t+2 \theta\left(1+|x(t)|^{2}\right)^{\theta-1} x^{T}(t) g(x(t), t) d B(t) .
$$

This implies

$$
\begin{aligned}
& \mathbb{E}\left(\sup _{t \leq u \leq t+1}\left(1+|x(u)|^{2}\right)^{\theta}\right) \leq \mathbb{E}\left[\left(1+|x(t)|^{2}\right)^{\theta}\right]+C_{1} \\
& \quad+2 \theta \mathbb{E}\left(\sup _{t \leq u \leq t+1}\left|\int_{t}^{u}\left(1+|x(s)|^{2}\right)^{\theta-1} x^{T}(s) g(x(s), s) d B(s)\right|\right) .
\end{aligned}
$$


But, by the well-known Burkholder-Davis-Gundy inequality (see e.g. [15, 17]), we compute

$$
\begin{aligned}
& 2 \theta \mathbb{E}\left(\sup _{t \leq u \leq t+1}\left|\int_{t}^{u}\left(1+|x(s)|^{2}\right)^{\theta-1} x^{T}(s) g(x(s), s) d B(s)\right|\right) . \\
\leq & 2 \theta \mathbb{E}\left(\int_{t}^{t+1}\left(1+|x(s)|^{2}\right)^{2 \theta-2}\left|x^{T}(s) g(x(s), s)\right|^{2} d s\right)^{\frac{1}{2}} \\
\leq & 2 \theta \mathbb{E}\left(\int_{t}^{t+1}\left(1+|x(s)|^{2}\right)^{2 \theta-2}|x(s)|^{2}\left(\eta+\gamma|x(t)|^{2}\right) d s\right)^{\frac{1}{2}} \\
\leq & 2 \theta \sqrt{\eta \vee \gamma \mathbb{E}}\left(\left[\sup _{t \leq s \leq t+1}\left(1+|x(s)|^{2}\right)^{\theta}\right] \int_{t}^{t+1}\left(1+|x(s)|^{2}\right)^{\theta} d s\right)^{\frac{1}{2}} \\
\leq & \frac{1}{2} \mathbb{E}\left[\sup _{t \leq s \leq t+1}\left(1+|x(s)|^{2}\right)^{\theta}\right]+2 \theta^{2}(\eta \vee \gamma) \mathbb{E} \int_{t}^{t+1}\left(1+|x(s)|^{2}\right)^{\theta} d s .
\end{aligned}
$$

Substituting this into (2.11) gives

$$
\begin{aligned}
\mathbb{E}\left(\sup _{t \leq u \leq t+1}\left(1+|x(u)|^{2}\right)^{\theta}\right) & \leq 2 \mathbb{E}\left[\left(1+|x(t)|^{2}\right)^{\theta}\right]+2 C_{1} \\
& +4 \theta^{2}(\eta \vee \gamma) \int_{t}^{t+1} \mathbb{E}\left(1+|x(s)|^{2}\right)^{\theta} d s .
\end{aligned}
$$

Letting $t \rightarrow \infty$ and using (2.10) we obtain that

$$
\limsup _{t \rightarrow \infty} \mathbb{E}\left(\sup _{t \leq u \leq t+1}\left(1+|x(u)|^{2}\right)^{\theta}\right) \leq 2 C_{1}\left[1+\frac{1}{\varepsilon}\left(1+4 \theta^{2}(\eta \vee \gamma)\right)\right] .
$$

Therefore, there is a positive constant $C_{2}$ such that

$$
\mathbb{E}\left(\sup _{k \leq u \leq k+1}|x(u)|^{2 \theta}\right) \leq C_{2}, \quad k=1,2, \cdots
$$

Let $\bar{\varepsilon}>0$ be arbitrary. Then, by the well-known Chebyshev inequality, we have

$$
\mathbb{P}\left\{\sup _{k \leq t \leq k+1}|x(u)|^{2 \theta}>k^{1+\bar{\varepsilon}}\right\} \leq \frac{C_{2}}{k^{1+\bar{\varepsilon}}}, \quad k=1,2, \cdots .
$$

Applying the well-known Borel-Cantelli lemma (see e.g. [15]), we obtain that for almost all $\omega \in \Omega$,

$$
\sup _{k \leq t \leq k+1}|x(t)|^{2 \theta} \leq k^{1+\bar{\varepsilon}}
$$

holds for all but finitely many $k$. Hence, there exists a $k_{0}(\omega)$, for almost all $\omega \in \Omega$, for which (2.13) holds whenever $k \geq k_{0}$. Consequently, for almost all $\omega \in \Omega$, if $k \geq k_{0}$ and $k \leq t \leq k+1$,

$$
\frac{\log \left(|x(t)|^{2 \theta}\right)}{\log t} \leq \frac{(1+\bar{\varepsilon}) \log k}{\log k}=1+\bar{\varepsilon}
$$

Therefore

$$
\limsup _{t \rightarrow \infty} \frac{\log (|x(t)|)}{\log t} \leq \frac{1+\bar{\varepsilon}}{2 \theta} \text { a.s. }
$$


Letting $\bar{\varepsilon} \rightarrow 0$ we obtain that

$$
\limsup _{t \rightarrow \infty} \frac{\log (|x(t)|)}{\log t} \leq \frac{1}{2 \theta} \quad \text { a.s. }
$$

Since this holds for any $\theta$ which obeys (2.6), we must have the desired assertion (2.5). The proof is therefore complete.

Let us now return to the ordinary differential equation (1.1) and its corresponding SDE (1.2). If we define

$$
f(x, t)=a+b x \quad \text { and } \quad g(x, t)=\sigma x, \quad(x, t) \in \mathbb{R} \times \mathbb{R}_{+}
$$

and let $B(t)$ be a scalar Brownian motion. Then equation (1.2) can be written as equation (2.1). In this case, we clearly have

$$
|g(x, t)|^{2}=\sigma^{2} x^{2} \quad \text { and } \quad|x g(x, t)|^{2}=\sigma^{2} x^{4} .
$$

Moreover, for any sufficiently small $\varepsilon>0$,

$$
x f(x, t)=a x+b x^{2} \leq \frac{2 a^{2}}{\varepsilon}+(b+\varepsilon) x^{2} .
$$

By Theorem 2.2, we see that the solution of equation (1.2) obeys

$$
\limsup _{t \rightarrow \infty} \frac{\log (|x(t)|)}{\log t} \leq \frac{\sigma^{2}}{\sigma^{2}-2(b+\varepsilon)} \quad \text { a.s. }
$$

Letting $\varepsilon \rightarrow 0$ yields

$$
\limsup _{t \rightarrow \infty} \frac{\log (|x(t)|)}{\log t} \leq \frac{\sigma^{2}}{\sigma^{2}-2 b} \quad \text { a.s. }
$$

which confirms (1.3).

\section{Noise Suppresses Exponential Growth}

Let us now consider a nonlinear system described by an ordinary differential equation

$$
\dot{y}(t)=f(y(t), t) .
$$

Here $f: \mathbb{R}^{n} \times \mathbb{R}_{+} \rightarrow \mathbb{R}^{n}$ is locally Lipschitz continuous and obeys

$$
\langle x, f(x, t)\rangle \leq \alpha+\beta|x|^{2}, \quad \forall(x, t) \in \mathbb{R}^{n} \times \mathbb{R}_{+},
$$

for some positive constants $\alpha$ and $\beta$. Clearly, the solution of this equation may grow exponentially. The question is: Can we design a linear stochastic feedback control of the form

$$
\sum_{i=1}^{m} A_{i} x(t) d B_{i}(t)
$$

(i.e. choose square matrices $A_{i} \in \mathbb{R}^{n \times n}$ ) so that the stochastically controlled system

$$
d x(t)=f(x(t), t) d t+\sum_{i=1}^{m} A_{i} x(t) d B_{i}(t)
$$

will grow at most polynomially with probability one? Let us first establish a corollary from Theorem 2.2. Based on this corollary, we will then answer the question very positively. 
Corollary 3.1 Let (3.2) hold. Assume that there are two positive constants $\gamma$ and $\delta$ such that

$$
\sum_{i=1}^{m}\left|A_{i} x\right|^{2} \leq \gamma|x|^{2}, \quad \sum_{i=1}^{m}\left|x^{T} A_{i} x\right|^{2} \geq \delta|x|^{4}
$$

for all $x \in \mathbb{R}^{n}$, and

$$
\delta>\beta+\frac{1}{2} \gamma
$$

Then, for any initial value $x(0)=x_{0} \in \mathbb{R}^{n}$, the solution of the stochastically controlled system (3.3) obeys

$$
\limsup _{t \rightarrow \infty} \frac{\log (|x(t)|)}{\log t} \leq \frac{\delta}{2 \delta-2 \beta-\gamma} \quad \text { a.s. }
$$

Proof. Define $g: \mathbb{R}^{n} \times \mathbb{R}_{+} \rightarrow \mathbb{R}^{n \times m}$ by

$$
g(x, t)=\left(A_{1} x, \cdots, A_{m} x\right) .
$$

Then system (3.3) can be written as equation (2.1). Moreover,

$$
|g(x, t)|^{2}=\sum_{i=1}^{m}\left|A_{i} x\right|^{2} \leq \gamma|x|^{2} \quad \text { and } \quad\left|x^{T} g(x, t)\right|^{2}=\sum_{i=1}^{m}\left|x^{T} A_{i} x\right|^{2} \geq \delta|x|^{4} .
$$

The conclusion hence follows from Theorem 2.2 directly.

The reader may wonder if the following more general stochastic feedback control

$$
\sum_{i=1}^{m}\left(a_{i}+A_{i} x(t)\right) d B_{i}(t)
$$

could be better? Here $a_{i} \in \mathbb{R}^{n}, 1 \leq i \leq n$. The answer is not. In fact, we can show in the same way as the above corollary was proved that under the same conditions of Corollary 3.1, the solution of the following SDE

$$
d x(t)=f(x(t), t) d t+\sum_{i=1}^{m}\left(a_{i}+A_{i} x(t)\right) d B_{i}(t)
$$

still obeys (3.6).

Let us now show that there are many matrices $A_{i}$ that satisfy conditions (3.4) and (3.5). First of all, let $A_{i}=\sigma_{i} I$ for $1 \leq i \leq m$, where $I$ is the $n \times n$ identity matrix and $\sigma_{i}$ 's are non-negative real numbers which represent the intensity of the noise. In this case, the stochastically controlled system becomes

$$
d x(t)=f(x(t), t) d t+\sum_{i=1}^{m} \sigma_{i} x(t) d B_{i}(t) .
$$

By Corollary 3.1, it is straightforward to show that the solution of system (3.7) obeys

$$
\limsup _{t \rightarrow \infty} \frac{\log (|x(t)|)}{\log t} \leq \frac{\sum_{i=1}^{m} \sigma_{i}^{2}}{\sum_{i=1}^{m} \sigma_{i}^{2}-2 \beta} \quad \text { a.s. }
$$


Hence, the solution will grow at most polynomially with probability one provided $\sum_{i=1}^{m} \sigma_{i}^{2}>$ $2 \beta$.

Let us consider a more general case. For each $i$, choose a positive-definite matrix $D_{i}$ such that

$$
x^{T} D_{i} x \geq \frac{\sqrt{3}}{2}\left\|D_{i}\right\||x|^{2} \quad \forall x \in \mathbb{R}^{n} .
$$

Obviously, there are many such matrices. Let $\sigma$ be a constant large enough for

$$
\sigma^{2}>\frac{4 \beta}{\sum_{i=1}^{m}\left\|D_{i}\right\|^{2}}
$$

Set $A_{i}=\sigma D_{i}$. Then

$$
\sum_{i=1}^{m}\left|A_{i} x\right|^{2} \leq \sigma^{2} \sum_{i=1}^{m}\left\|D_{i}\right\|^{2}|x|^{2} \quad \text { and } \quad \sum_{i=1}^{m}\left|x^{T} A_{i} x\right|^{2} \geq \frac{3 \sigma^{2}}{4} \sum_{i=1}^{m}\left\|D_{i}\right\|^{2}|x|^{4} .
$$

Thus, by Corollary 3.1, we can conclude that the solution of the stochastically controlled system (3.3) obeys

$$
\limsup _{t \rightarrow \infty} \frac{\log (|x(t)|)}{\log t} \leq \frac{\frac{3 \sigma^{2}}{4} \sum_{i=1}^{m}\left\|D_{i}\right\|^{2}}{\frac{\sigma^{2}}{2} \sum_{i=1}^{m}\left\|D_{i}\right\|^{2}-2 \beta} \quad \text { a.s. }
$$

Summarizing these cases we obtain the following result.

Theorem 3.2 The potentially exponential growth of the solution to a nonlinear system $\dot{y}(t)=f(y(t), t)$ can be suppressed by Brownian motions provided (3.2) is satisfied. Moreover, one can even use only a scalar Brownian motion to suppress the exponential growth.

In particular, given any linear ordinary differential equation

$$
\frac{d y(t)}{d t}=q+Q y(t), \quad t \geq 0
$$

where $q \in \mathbb{R}^{n}$ and $Q \in \mathbb{R}^{n \times n}$, we may stochastically perturb it into the linear SDE

$$
d x(t)=(q+Q x(t)) d t+\sum_{i=1}^{m} A_{i} x(t) d B_{i}(t)
$$

where $A_{i}=\sigma D_{i}, D_{i}$ 's obey (3.8) and $\sigma>0$ is large enough for

$$
\sigma^{2}>\frac{4\|Q\|}{\sum_{i=1}^{m}\left\|D_{i}\right\|^{2}}
$$

Note that for any sufficiently small $\varepsilon>0$,

$$
\langle x, q+Q x\rangle \leq|q||x|+\left.\|Q\||| x\right|^{2} \leq \frac{2|q|^{2}}{\varepsilon}+(\|Q\|+\varepsilon)|x|^{2} .
$$


Using this and (3.9) we observe, by Corollary 3.1, that the solution of the linear controlled system (3.10) obeys

$$
\limsup _{t \rightarrow \infty} \frac{\log (|x(t)|)}{\log t} \leq \frac{\frac{3 \sigma^{2}}{4} \sum_{i=1}^{m}\left\|D_{i}\right\|^{2}}{\frac{\sigma^{2}}{2} \sum_{i=1}^{m}\left\|D_{i}\right\|^{2}-2(\|Q\|+\varepsilon)} \quad \text { a.s. }
$$

Since $\varepsilon>0$ is arbitrary, we can therefore conclude that under conditions (3.8) and (3.11), the solution of the linear controlled system (3.10) obeys

$$
\limsup _{t \rightarrow \infty} \frac{\log (|x(t)|)}{\log t} \leq \frac{\frac{3 \sigma^{2}}{4} \sum_{i=1}^{m}\left\|D_{i}\right\|^{2}}{\frac{\sigma^{2}}{2} \sum_{i=1}^{m}\left\|D_{i}\right\|^{2}-2\|Q\|} \quad \text { a.s. }
$$

\section{Exponential Growth of SDEs}

We have just shown that noise can suppress exponential growth. However, every thing has two sides. We may therefore wonder if noise can make a given system whose solutions are bounded become a new system whose solutions will grow exponentially? For example, consider the $n$-dimensional linear ordinary differential equation

$$
\frac{d y(t)}{d t}=q+Q y(t), \quad t \geq 0
$$

with initial value $y(0)=y_{0} \in \mathbb{R}^{n}$, where $q \in \mathbb{R}^{n}$ and $Q \in \mathbb{R}^{n \times n}$. Assume that

$$
-\lambda:=\lambda_{\max }\left(Q+Q^{T}\right)<0
$$

Equation (4.2) can be solved explicitly, which implies easily that

$$
\limsup _{t \rightarrow \infty}|y(t)| \leq|q|
$$

That is, under condition (4.2), the solution of equation (4.1) is asymptotically bounded and the bound is independent of the initial value. The question is: Can we stochastically perturb this equation into an Itô SDE

$$
d x(t)=(q+Q x(t)) d t+g(x(t), t) d B(t)
$$

so that its solutions will grow exponentially with probability one? Moreover, can the noise term $g(x(t), t) d B(t)$ be designed to be a linear form of $x(t)$ ?

To answer these questions, let us first establish a general result on the exponential growth of the solutions to stochastic differential equations. As a standing hypothesis in this section, we assume that the coefficients $f$ and $g$ are smooth enough so that equation (2.1) has a unique global solution.

Theorem 4.1 Assume that there are non-negative constants $c_{1}-c_{6}$ such that

$$
\begin{gathered}
c_{5}>c_{1}, \quad c_{6}>c_{2}+2 c_{4}, \\
-2\langle x, f(x, t)\rangle \leq c_{1}+c_{2}|x|^{2}, \quad\left|x^{T} g(x, t)\right|^{2} \leq c_{3}|x|^{2}+c_{4}|x|^{4}
\end{gathered}
$$


and

$$
|g(x, t)|^{2} \geq c_{5}+c_{6}|x|^{2}
$$

for all $(x, t) \in \mathbb{R}^{n} \times \mathbb{R}_{+}$. Set

$$
a=c_{5}-c_{1}, \quad b=c_{6}-c_{2}-2 c_{4}, \quad c=c_{5}-c_{1}+c_{6}-c_{2}-2 c_{3} .
$$

(i) If, furthermore,

$$
c \geq 2(a \wedge b),
$$

then the solution of equation (2.1) obeys

$$
\liminf _{t \rightarrow \infty} \frac{1}{t} \log (|x(t)|) \geq \frac{1}{2}(a \wedge b) \quad \text { a.s. }
$$

(ii) If (4.9) does not hold but

$$
a b>\frac{1}{4} c^{2},
$$

then the solution of equation (2.1) obeys

$$
\liminf _{t \rightarrow \infty} \frac{1}{t} \log (|x(t)|) \geq \frac{1}{2} \min \left\{a, b, \frac{a b-0.25 c^{2}}{a+b-c}\right\} \quad \text { a.s. }
$$

Proof. By the Itô formula and conditions (4.6) and (4.7), we compute

$$
\begin{aligned}
d\left[\log \left(1+|x(t)|^{2}\right)\right] & =\left(\frac{2\langle x(t), f(x(t), t)\rangle+|g(x(t), t)|^{2}}{1+|x(t)|^{2}}-\frac{2\left|x^{T}(t) g(x(t), t)\right|^{2}}{\left(1+|x(t)|^{2}\right)^{2}}\right) d t \\
& -\frac{2 x^{T}(t) g(x(t), t)}{1+|x(t)|^{2}} d B(t) \\
& \geq\left(\frac{\left(c_{5}-c_{1}\right)+\left(c_{6}-c_{2}\right)|x(t)|^{2}}{1+|x(t)|^{2}}-\frac{2 c_{3}|x(t)|^{2}+2 c_{4}|x(t)|^{4}}{\left(1+|x(t)|^{2}\right)^{2}}\right) d t \\
& +\frac{2 x^{T}(t) g(x(t), t)}{1+|x(t)|^{2}} d B(t) \\
& =\frac{F\left(|x(t)|^{2}\right)}{\left(1+|x(t)|^{2}\right)^{2}} d t+\frac{2 x^{T}(t) g(x(t), t)}{1+|x(t)|^{2}} d B(t),
\end{aligned}
$$

where $F: \mathbb{R}_{+} \rightarrow \mathbb{R}$ is defined by

$$
F(u)=a+c u+b u^{2},
$$

in which the parameters $a, b$ and $c$ have been defined by (4.8). Let us now consider the two cases:

Case (i): By condition (4.9), we have

$$
F(u) \geq(a \wedge b)(1+u)^{2} \quad \text { on } u \geq 0 .
$$

Hence

$$
\frac{F\left(|x(t)|^{2}\right)}{\left(1+|x(t)|^{2}\right)^{2}} \geq(a \wedge b) .
$$


It therefore follows from (4.13) that

$$
\log \left(1+|x(t)|^{2}\right) \geq \log \left(1+\left|x_{0}\right|^{2}\right)+(a \wedge b) t+M(t),
$$

where

$$
M(t)=\int_{0}^{t} \frac{2 x^{T}(t) g(x(t), t)}{1+|x(t)|^{2}} d B(t)
$$

which is a continuous martingale with initial value $M(0)=0$. By condition (4.6), we compute its quadratic variation

$$
\langle M(t)\rangle=\int_{0}^{t} \frac{4\left|x^{T}(t) g(x(t), t)\right|}{1+|x(t)|^{2}} d t \leq \int_{0}^{t} \frac{4\left(c_{3}|x(t)|^{2}+c_{4}|x(t)|^{4}\right.}{1+|x(t)|^{2}} d t \leq 4\left(c_{3}+c_{4}\right) t .
$$

Hence, by the strong law of large numbers of martingales (see e.g. [15, Theorem 3.4 on page 12]),

$$
\lim _{t \rightarrow \infty} \frac{M(t)}{t}=0 \quad \text { a.s. }
$$

Dividing both sides of (4.14) and then letting $t \rightarrow \infty$ we then obtain

$$
\liminf _{t \rightarrow \infty} \frac{1}{t} \log \left(1+|x(t)|^{2}\right) \leq(a \wedge b) \quad \text { a.s. }
$$

which yields the required assertion (4.10) immediately.

Case (ii): Under condition (4.11) we now look for a positive number $\lambda$ for

$$
F(u) \geq \lambda(1+u)^{2} \quad \forall u \geq 0 .
$$

Write

$$
\begin{aligned}
F(u)-\lambda(1+u)^{2} & =a-\lambda+(c-2 \lambda) u+(b-\lambda) u^{2} \\
& =(1, u)\left(\begin{array}{cc}
a-\lambda & 0.5(c-2 \lambda) \\
0.5(c-2 \lambda) & b-\lambda
\end{array}\right)\left(\begin{array}{l}
1 \\
u
\end{array}\right) .
\end{aligned}
$$

It is therefore clear that (4.17) will hold if

$$
\lambda \leq a \wedge b \quad \text { and } \quad(a-\lambda)(c-\lambda) \geq 0.25(c-2 \lambda)^{2},
$$

namely

$$
\lambda \leq a \wedge b \quad \text { and } \quad(a+b-c) \lambda \leq a b-0.25 c^{2} .
$$

As (4.9) does not hold, we must have $c<2(a \wedge b) \leq a+b$. We can therefore choose the positive number

$$
\lambda=\min \left\{a, b, \frac{a b-0.25 c^{2}}{a+b-c}\right\}
$$

for (4.17) to hold. Hence

$$
\frac{F\left(|x(t)|^{2}\right)}{\left(1+|x(t)|^{2}\right)^{2}} \geq \lambda
$$

It therefore follows from (4.13) that

$$
\log \left(1+|x(t)|^{2}\right) \geq \log \left(1+\left|x_{0}\right|^{2}\right)+\lambda t+M(t)
$$


where $M(t)$ is the same as before. This, together with (4.15), implies

$$
\liminf _{t \rightarrow \infty} \frac{1}{t} \log \left(1+|x(t)|^{2}\right) \geq \lambda \quad \text { a.s. }
$$

and hence the assertion (4.12) follows.

Let us make some comments on the conditions of Theorem 4.1. First of all, we observe that condition (4.6) can be satisfied by a large class of functions. For example, if both $f$ and $g$ obey the linear growth condition

$$
|f(x, t)| \vee|g(x, t)| \leq K(1+|x|),
$$

then

$$
-2\langle x, f(x, t)\rangle \leq 2|x||f(x, t)| \leq 2 K|x|+2 K|x|^{2} \leq K+3 K|x|^{2}
$$

and

$$
\left|x^{T} g(x, t)\right|^{2} \leq|x|^{2}|g(x, t)|^{2} \leq K^{2}|x|^{2}(1+|x|)^{2} \leq 2 K^{2}\left(|x|^{2}+|x|^{4}\right),
$$

that is $f$ and $g$ obey (4.6). In particular, linear SDEs always obey (4.6). However, as shown in the previous section, there are many linear SDEs whose solutions will grow at most polynomially but not exponentially with probability one. This of course indicates that condition (4.7) is very critical in order to have an exponential growth. Nevertheless, instead of using the linear growth condition, the forms described in (4.6) enable us to compute the parameters $c_{1}-c_{4}$ more precisely in many situations as illustrated in the examples discussed in next section.

\section{Noise Expresses Exponential Growth}

Let us now begin to answers the questions asked in the beginning of the previous section. Consider an $n$-dimensional ordinary differential equation

$$
\frac{d y(t)}{d t}=f(y(t), t), \quad t \geq 0
$$

Assume that $f$ is sufficiently smooth and, in particular, it obeys

$$
-2\langle x, f(x, t)\rangle \leq c_{1}+c_{2}|x|^{2}, \quad \forall(x, t) \in \mathbb{R}^{n} \times \mathbb{R}
$$

for some non-negative numbers $c_{1}$ and $c_{2}$. Our aim here is to perturb this equation stochastically into an SDE

$$
d x(t)=f(x(t), t) d t+g(x(t)) d B(t)
$$

so that its solutions will grow exponentially with probability one. We will design $g$ to be independent of $t$ so we write $g(x, t)$ as $g(x)$ in this section. Moreover, we shall see that the noise term $g(x(t)) d B(t)$ can be designed to be a linear form of $x(t)$.

First, let the dimension of the state space $n$ be an even number and choose the dimension of the Brownian motion $m$ to be 2 . Design $g: \mathbb{R}^{n} \rightarrow \mathbb{R}^{n \times 2}$ by

$$
g(x)=(\xi, A x)
$$


where $\xi=\left(\xi_{1}, \cdots, \xi_{n}\right)^{T} \in \mathbb{R}^{n}$ and, of course, $\xi \neq 0$, while

$$
A=\left(\begin{array}{ccccc}
0 & \sigma & & & \\
-\sigma & 0 & & & \\
& & \ddots & & \\
& & & 0 & \sigma \\
& & & -\sigma & 0
\end{array}\right)
$$

with $\sigma>0$. So the stochastically perturbed system (5.3) becomes

$$
d x(t)=f(x(t), t) d t+\xi d B_{1}+\sigma\left(\begin{array}{c}
x_{2}(t) \\
-x_{1}(t) \\
\vdots \\
x_{n}(t) \\
-x_{n-1}(t)
\end{array}\right) d B_{2}(t) .
$$

For $x \in \mathbb{R}^{n}$, compute

$$
\left|x^{T} g(x)\right|^{2}=\left(x^{T} \xi\right)^{2}+\left(x^{T} A x\right)^{2}=\left(x^{T} \xi\right)^{2} \leq|\xi|^{2}|x|^{2}
$$

and

$$
|g(x)|^{2}=|\xi|^{2}+|A x|^{2}=|\xi|^{2}+\sigma^{2}|x|^{2} .
$$

That is, conditions (4.6) and (4.7) are satisfied with

$$
c_{3}=|\xi|^{2}, \quad c_{4}=0, \quad c_{5}=|\xi|^{2}, \quad c_{6}=\sigma^{2} .
$$

Consequently, the parameters defined by (4.8) becomes

$$
a=|\xi|^{2}-c_{1}, \quad b=\sigma^{2}-c_{2}, \quad c=\sigma^{2}-c_{1}-c_{2}-|\xi|^{2} .
$$

If we choose

$$
|\xi|^{2}>c_{1} \quad \text { and } \quad \sigma^{2}=c_{1}+c_{2}+|\xi|^{2}
$$

then $b=|\xi|^{2}+c_{1} \geq a, c=0$ and

$$
\frac{a b-0.25 c^{2}}{a+b-c}=\frac{|\xi|^{4}-c_{1}^{2}}{2|\xi|^{2}} \leq \frac{a\left(|\xi|^{2}+c_{1}\right)}{2|\xi|^{2}}<a,
$$

and hence, by Theorem 4.1, the solution of equation (5.4) obeys

$$
\liminf _{t \rightarrow \infty} \frac{1}{t} \log (|x(t)|) \geq \frac{|\xi|^{2}-c_{1}^{2}}{4|\xi|^{2}} \quad \text { a.s. }
$$

Alternatively, if we choose

$$
|\xi|^{2}>c_{1} \quad \text { and } \quad \sigma^{2}=3|\xi|^{2}+c_{2}-c_{1}
$$

then $b=3|\xi|^{2}-c_{1}=2|\xi|^{2}+a>a, c=2 a$ and hence, by Theorem 4.1, the solution of equation (5.4) obeys

$$
\liminf _{t \rightarrow \infty} \frac{1}{t} \log (|x(t)|) \geq \frac{1}{2}\left(|\xi|^{2}-c_{1}\right) \quad \text { a.s. }
$$


We next let the dimension of the state space $n$ be an odd number but $n \geq 3$. Choose the dimension of the Brownian motion $m$ to be 3 and design $g: \mathbb{R}^{n} \rightarrow \mathbb{R}^{n \times 3}$ by

$$
g(x)=\left(\xi, A_{2} x, A_{3} x\right)
$$

where $\xi=\left(\xi_{1}, \cdots, \xi_{n}\right)^{T} \in \mathbb{R}^{n}$ and, of course, $\xi \neq 0$, while

$$
A_{2}=\left(\begin{array}{cccccc}
0 & \sigma & & & & \\
-\sigma & 0 & & & & \\
& & \ddots & & & \\
& & & 0 & \sigma & \\
& & & -\sigma & 0 & \\
& & & & & 0
\end{array}\right) \text { and } A_{3}=\left(\begin{array}{cccccc}
0 & & & & & \\
& 0 & \sigma & & & \\
& -\sigma & 0 & & & \\
& & & \ddots & & \\
& & & 0 & \sigma \\
& & & -\sigma & 0
\end{array}\right)
$$

with $\sigma>0$. So the stochastically perturbed system (5.3) becomes

$$
d x(t)=f(x(t), t) d t+\xi d B_{1}+\sigma\left(\begin{array}{c}
x_{2}(t) \\
-x_{1}(t) \\
\vdots \\
x_{n-1}(t) \\
-x_{n-2}(t) \\
0
\end{array}\right) d B_{2}(t)+\sigma\left(\begin{array}{c}
0 \\
x_{2}(t) \\
-x_{3}(t) \\
\vdots \\
x_{n}(t) \\
-x_{n-1}(t)
\end{array}\right) d B_{3}(t) .
$$

For $x \in \mathbb{R}^{n}$, compute

$$
\left|x^{T} g(x)\right|^{2}=\left(x^{T} \xi\right)^{2}+\left(x^{T} A_{2} x\right)^{2}+\left(x^{T} A_{3} x\right)^{2}=\left(x^{T} \xi\right)^{2} \leq|\xi|^{2}|x|^{2}
$$

and

$$
|g(x)|^{2}=|\xi|^{2}+\left|A_{2} x\right|^{2}+\left|A_{3} x\right|^{2} \geq|\xi|^{2}+\sigma^{2}|x|^{2} .
$$

Hence, conditions (4.6) and (4.7) are satisfied with the same parameters specified by (5.5). Hence, as shown above, if we choose $\xi$ and $\sigma$ as (5.6) then the solution of equation (5.10) obeys (5.7), while if we choose $\xi$ and $\sigma$ as (5.8) then the solution of equation (5.10) obeys (5.9).

Summarizing the above arguments, we obtain the following result.

Theorem 5.1 Any nonlinear system $\dot{y}(t)=f(y(t), t)$ can be stochastically perturbed by Brownian motions into the SDE (5.3) whose solutions will grow exponentially with probability one provided (5.2) is satisfied and the dimension of the state space is greater than 1. Moreover, the noise term $g(x(t)) d B(t)$ in (5.3) can be designed to be a linear form of $x(t)$.

In this theorem we require the dimension of the state space be greater than 1 . Naturally, we may wonder what happens in the scalar case? More precisely, the question is: Given a scalar system $\dot{y}(t)=f(y(t), t)$ whose solutions are bounded, can we stochastically perturb it into an SDE whose solutions will grow exponentially with probability one? To answer this question, let us consider a linear ordinary differential equation

$$
\dot{y}(t)=p-q y(t), \quad t \geq 0,
$$


where $p$ and $q$ are both positive numbers. It is known that for any given initial value, the solution of this equation obeys

$$
\lim _{t \rightarrow \infty} y(t)=\frac{p}{q} .
$$

In other words, the solution is asymptotically bounded. Let us now consider the corresponding linear SDE

$$
d x(t)=(p-q x(t)) d t+(u+v x(t)) d B(t)
$$

where both $u$ and $v$ are positive numbers while $B(t)$ is a scalar Brownian motion. By defining $f(x, t)=p-q x$ and $g(x, t)=u+v x$ for $(x, t) \in \mathbb{R} \times \mathbb{R}_{+}$, equation (5.11) becomes the form of (2.1). Compute

$$
x f(x, t)=p x-q x^{2} \leq \frac{p^{2}}{4 q} .
$$

Also, for any sufficiently small $\varepsilon>0$,

$$
|g(x, t)|^{2}=u^{2}+2 u v x+v^{2} x^{2} \leq u^{2}+\frac{u^{2} v^{2}}{\varepsilon}+\left(v^{2}+\varepsilon\right) x^{2}
$$

and

$$
|x g(x, t)|^{2}=u^{2} x^{2}+2 u v x^{3}+v^{2} x^{4} \geq\left(v^{2}-\varepsilon\right) x^{4}-\rho,
$$

where $-\rho$ is a lower bound of $u^{2} x^{2}+2 u v x^{3}+\varepsilon x^{4}$ on $x \in \mathbb{R}$. By Theorem 2.2, we see that the solution of equation (5.12) obeys

$$
\limsup _{t \rightarrow \infty} \frac{\log (|x(t)|)}{\log t} \leq \frac{v^{2}-\varepsilon}{v^{2}-3 \varepsilon} \quad \text { a.s. }
$$

Letting $\varepsilon \rightarrow 0$ we conclude that the solution of equation (5.12) obeys

$$
\limsup _{t \rightarrow \infty} \frac{\log (|x(t)|)}{\log t} \leq 1 \quad \text { a.s. }
$$

That is, the solution of equation (5.12) will grow at most polynomially with probability one. Similarly, we can show that the solution to the following more general linear SDE

$$
d x(t)=(p-q x(t)) d t+\sum_{i=1}^{m}\left(u_{i}+v_{i} x(t)\right) d B_{i}(t)
$$

also obeys (5.13). In other words, we have shown that the linear stochastic perturbation $\sum_{i=1}^{m}\left(u_{i}+v_{i} x(t)\right) d B_{i}(t)$ may not force the solution of a scalar system $\dot{y}(t)=f(y(t), t)$ to grow exponentially.

\section{Acknowledgements}

The authors would like to thank the referees for their very helpful comments and suggestions. The authors would also like to thank the EPSRC (U.K.) (grant EP/E009409), the National Natural Science Foundation of China (grants 60574069, 60574042 and 60374023), the Key Programs of Science and Technology of Guangzhou (grant 2007Z3-D0171 ) as well as Jinan University in China for their financial support. 


\section{References}

[1] Appleby, J.A.D., Stabilisation of functional differential equations by noise, Systems Control Lett., to appear.

[2] Appleby, J.A.D., Destabilisation of functional differential equations by noise, Systems Control Lett., to appear.

[3] Appleby, J.A.D. and Flynn, A., Stabilisation of Volterra equations by noise, J. Appl. Math. Stoch. Anal., to appear.

[4] Appleby, J.A.D. and Mao, X., Stochastic stabilisation of functional differential equations, System Control Lett. 54(11) (2005), 1069-1081.

[5] Appleby, J.A.D., Mao, X. and Rodkina, A., On stochastic stabilisation of difference equations, Discrete and Continuous Dynamical Systems-Series A 15(3) (2006), 843857.

[6] Arnold, L., Random Dynamical Systems, Springer, 1998.

[7] Arnold, L., Crauel, H. and Wihstutz, V., Stabilisation of linear systems by noise, SIAM J. Control Optim. 21 (1983), 451-461.

[8] Bellman, R., Bentsman, J. and Meerkov, S., Stability of fast periodic systems, IEEE Tans. Automa. Control 30 (1985), 289-291.

[9] Caraballo, T., Garrido-Atienza, M. and Real, J. Stochastic stabilisation of differential systems with general decay rate. Systems Control Lett., 48 (2003), 397-406.

[10] Hasminskii, R.Z., Stochastic Stability of Differential Equations, Sijthoff and Noordhoff, 1981.

[11] Kushner, H. J., On the stability of processes defined by stochastic differencedifferential equations, J. Differential Equations 4 (1968), 424-443.

[12] Mao, X., Stability of Stochastic Differential Equations with Respect to Semimartingales, Longman Scientific and Technical, 1991.

[13] Mao, X., Exponential Stability of Stochastic Differential Equations, Marcel Dekker, 1994.

[14] Mao, X., Stochastic stabilisation and destabilisation, Systems and Control Letters 23 (1994), 279-290.

[15] Mao, X., Stochastic Differential Equations and Their Applications, Chichester: Horwood Pub., 1997.

[16] Mao, X., Marion, G. and Renshaw, E., Environmental noise suppresses explosion in population dynamics, Stochastic Processes and Their Applications 97 (2002), 95-110.

[17] Mao, X. and Yuan, C., Stochastic Differential Equations with Markovian Switching, Imperial College Press, 2006. 
[18] Meerkov, S., Condition of vibrational stabilizability for a class of non-linear systems, IEEE Trans. Automat. Control 27 (1982), 485-487.

[19] Pinsky, M.A. and Wihstutz, V., Lyapunov exponents of nilponent Itô systems, Stochastics 25 (1988), 43-57.

[20] Scheutzow, M., Stabilisation and destabilisation by noise in the plane, Stoch. Anal. Appl. 11(1) (1993), 97-113.

[21] Zhabko, A.P. and Kharitonov, V.L., Problem of vibrational stabilisation of linear systems, Automat. Telemekh. 2 (1980), 31-34. 